\title{
Network analysis of depressive symptoms in Hong Kong residents during the COVID-19 pandemic
}

\author{
Teris Cheung ${ }^{1,27 凶}$, Yu Jin (iD ${ }^{2,27}$, Simon Lam (iD ${ }^{1}$, Zhaohui Su ID $^{3}$, Brian J. Hall ${ }^{4,5}$, Yu-Tao Xiang ${ }^{6,7,8}$ and the International Research \\ Collaboration on COVID-19*
}

(c) The Author(s) 2021

In network theory depression is conceptualized as a complex network of individual symptoms that influence each other, and central symptoms in the network have the greatest impact on other symptoms. Clinical features of depression are largely determined by sociocultural context. No previous study examined the network structure of depressive symptoms in Hong Kong residents. The aim of this study was to characterize the depressive symptom network structure in a community adult sample in Hong Kong during the COVID-19 pandemic. A total of 11,072 participants were recruited between 24 March and 20 April 2020. Depressive symptoms were measured using the Patient Health Questionnaire-9. The network structure of depressive symptoms was characterized, and indices of "strength", "betweenness", and "closeness" were used to identify symptoms central to the network. Network stability was examined using a case-dropping bootstrap procedure. Guilt, Sad Mood, and Energy symptoms had the highest centrality values. In contrast, Concentration, Suicide, and Sleep had lower centrality values. There were no significant differences in network global strength $(p=0.259)$, distribution of edge weights $(p=0.73)$ and individual edge weights (all $p$ values $>0.05$ after Holm-Bonferroni corrections) between males and females. Guilt, Sad Mood, and Energy symptoms were central in the depressive symptom network. These central symptoms may be targets for focused treatments and future psychological and neurobiological research to gain novel insight into depression.

Translational Psychiatry (2021)11:460; https://doi.org/10.1038/s41398-021-01543-z

\section{INTRODUCTION}

Depression is a common psychiatric problem characterized by a range of symptoms such as low mood, guilt, and worthlessness [1]. Individuals with depression may experience immense personal and familial suffering, and they may also have other adverse outcomes such as impaired functioning, insomnia, economic burdens and even suicide [2-5]. In order to reduce the risk of depression and provide timely and effective treatments, it is important to understand the psychopathological mechanisms involved in depression.

In traditional theory of psychopathology (i.e., the common cause perspective of mental disorders) psychiatric symptoms are secondary to an underlying common cause [6, 7]. For instance, depression causes low mood, sleep disturbances and suicidality in the same fashion that infection causes fever and pain. Additionally, many studies used standardized scales on depression which sum individual item responses to generate a total score, which implies that depressive symptoms are viewed as interchangeable presentations of the same disorder [8]. However, evidence showed that individual depressive symptoms had different negative outcomes, risk factors and neurological mechanisms $[9,10]$. In clinical practice one depressive symptom may predict changes of other symptoms following treatments [11].

In a recently developed theory of psychopathology (i.e., the causal system perspective of mental disorders [12]), the cluster of co-occurring symptoms of depression is secondary to direct symptom-to-symptom relationships, but not a common cause. Accordingly, for example, low mood, anhedonia, and sleep disturbances were not caused by depression. Instead, they could affect each other with their own biological and psychological mechanisms. These symptom-symptom interactions can be estimated using network analysis. Central symptoms within this network exhibit the strongest association with many other symptoms. In addition, since central symptoms may activate other symptoms, they may play a major role in causing the onset of and/or maintaining a syndrome. Thus, targeting central symptoms with biopsychosocial interventions may be more efficient [13]. In the network theory, symptoms can be activated by their neighbor symptoms, or by external factors such as adverse life events, or medical diseases [14]. Network analysis also enables researchers to identify "bridge

\footnotetext{
${ }^{1}$ School of Nursing, Hong Kong Polytechnic University, Hong Kong SAR, China. ${ }^{2}$ College of Education for the Future, Beijing Normal University, Beijing, China. ${ }^{3}$ Center on Smart and Connected Health Technologies, Mays Cancer Center, School of Nursing, UT Health San Antonio, San Antonio, TX, USA. ${ }^{4}$ Global and Community Mental Health Research Group, New York University (Shanghai), Shanghai, China. ${ }^{5}$ School of Global Public Health, New York University, New York, NY, USA. ${ }^{6}$ Unit of Psychiatry, Department of Public Health and Medicinal Administration, Institute of Translational Medicine, Faculty of Health Sciences, University of Macau, Macao SAR, China. ${ }^{7}$ Centre for Cognitive and Brain Sciences, University of Macau, Macao SAR, China. ${ }^{8}$ Institute of Advanced Studies in Humanities and Social Sciences, University of Macau, Macao SAR, China. ${ }^{27}$ These authors contributed equally: Teris Cheung, Yu Jin. ${ }^{*} A$ list of authors and their affiliations appears at the end of the paper. ${ }^{凶}$ email: teris.cheung@polyu.edu.hk; xyutly@gmail.com
}

Received: 7 April 2021 Revised: 12 July 2021 Accepted: 26 July 2021

Published online: 06 September 2021 
symptoms" that mediate the transition among different syndromes [15-17].

Network analysis has been used to examine depressive symptoms in certain Western countries $[18,19]$. Evidence showed that patterns and clinical features of depression is greatly determined by socioeconomic contexts $[20,21]$, therefore symptom network structure of depression across different countries and socioeconomic backgrounds should be examined separately. Most studies on network structure of depression were conducted in the West, therefore the findings cannot be generalized in Asian settings including Hong Kong. More importantly, to date, only a paucity of studies utilized the network analysis to examine symptoms and symptom-to-symptom relationships in depression during the COVID-19 pandemic, despite depression having a high prevalence of psychiatric comorbidity in many populations [22]. This gave us the impetus to conduct this study to characterize the network structure of depressive symptoms in a large community adult sample in Hong Kong.

\section{METHODS \\ Settings and participants}

This large-scale, cross-sectional study was conducted in Hong Kong between 24 March and 20 April 2020 using snowball convenience sampling. The questionnaire was designed using Google form and Qualtrics, and delivered to several online platforms (e.g., WhatsApp, WeChat, Facebook). To be eligible, participants were Hong Kong residents who lived in Hong Kong during the COVID-19 pandemic, aged between 18 and 59 years and were able to read Chinese. This study protocol was approved by the Human Subjects Ethics Sub-committee of the Hong Kong Polytechnic University, Hong Kong (reference number: HSEARS20200227002-01). Participants provided their electronic informed consent prior to participation in this study. Participants were assured of their anonymity and confidentiality, and their rights of withdrawal were respected. Given the sensitive nature of some of the questions, a professional helpline directory was provided to participants.

\section{Measurements}

Depressive symptoms were measured by the Chinese version of the selfreport nine-item Patient Health Questionnaire (PHQ-9) [23] that measured a variety of cognitive, emotional, physiological and interpersonal symptoms of depression, such as Anhedonia, Sad Mood, Sleep, Energy, Appetite, Guilt, Concentration, Motor, and Suicide thoughts in the past two weeks [24]. Each item scored from 0 (not at all) to 3 (nearly every day), with a higher score indicating more severe depressive symptoms. The PHQ-9 has been well-validated in the Chinese populations [25-27].

\section{Network estimation}

The mean, standard deviation (SD), skewness and kurtosis of all the PHQ-9 items were calculated. Due to the controversy on the optimal method of model trichotomous items in network analysis [8], the values of all the PHQ-9 items were dichotomized as " 0 " and " 1 ", representing the absence and presence of depressive symptoms, respectively. Item values reporting " 0 " were transformed to absence of the symptom, whereas values of " 1,2 , or 3 " were transformed to presence of the symptom. The network model was estimated using the Ising model [28].

In the network analysis each individual depressive symptom was defined as "node" and relationships between these symptoms were "edges". For network visualization, the thickness of edges represented the strength of associations between nodes. The color of the edge indicated the direction of the correlations (e.g., green edges represented positive correlations; red edges represented negative correlations) [29].

The Ising model was used to assess network structures based on binary data $[28,30]$, which can be conceived as a series of pairwise associations among binary variables, after controlling for the confounding effects of all other associations. This method combined logistic regression with model selection based on a Goodness-of-Fit measure to identify relationships between nodes. To reduce the number of spurious edges and improve the interpretability of networks, the network models were regularized using the enhanced least absolute shrinkage and selection operator (eLASSO) [31]. This algorithm produced a sparse network model which became more interpretable than the original one. Model selection was based on the extend Bayesian Information Criterion (EBIC) [32]. The binary network was fitted using the R-package IsingFit 0.3.1 [28].

Three major centrality indices (i.e. Betweenness, Closeness, and Strength) were computed to examine which symptoms were most important in the depressive symptom network [33, 34]. Strength was used to measure the absolute sum of edge weights connected to a node, which indicated the importance of a particular factor. Betweenness was calculated by the frequency of a node lying on all the shortest paths between other nodes, while Closeness referred to the inverse of sum of distance from a node to all other nodes in the network [13]. The $R$ packages "IsingFit" "networktools" and "qgraph" in R program (version 3.6.3) [35] were used to perform the analyses [28, 29].

\section{Estimation of network accuracy and stability}

To assess the robustness of the results, we examined the accuracy and stability of the network model with three procedures [36]. First, the accuracy of edge-weights was estimated by computing confidence intervals (Cls) with non-parametric bootstrapping method [37]. Then, the observations in the data were resampled randomly to create new datasets from which the $95 \%$ Cls were calculated. Larger Cls suggested reduced precision in the estimation of the edges, and narrower Cls indicated a more trustworthy network [36].

Second, the correlation stability coefficient (CS-C) was used to assess the stability of centrality indices (i.e. Betweenness, Closeness, and Strength) using subset bootstraps [38]. If centrality indices of nodes did not change significantly after excluding part of the sample in the dataset, the network structure could be considered stable. The CS-C represented the maximum proportion of samples that could be removed, such that with $95 \%$ probability the correlation between original centrality indices could reach at least 0.7 [36]. Generally, the CS-C should not be less than 0.25 , and preferably above 0.5 . Then, the difference between two strength indices was considered significant if 1000-bootstrap 95\% non-parametric Cls did not contain zero.

Third, bootstrapped difference tests were used to evaluate differences in the network's properties [36]. This test relied on $95 \% \mathrm{Cls}$, to determine if two edge-weights or two node centrality indices significantly differed from one-another. The R package "bootnet" was used to perform the analyses [39].

\section{Association between symptom mean levels, variability, and centrality index}

Spearman's rank-order correlation was calculated between centrality indexes and the mean PHQ-9 item scores, and between centrality indexes and standard deviation for symptoms [18]. The correlation between centrality indexes and the mean PHQ-9 item scores was used to test whether the most central symptoms are the most severe ones, while the correlation between centrality indexes and standard deviation was used to test whether symptom centrality could be attributed to the items' differential variability [9].

\section{Comparison of network characteristics by gender}

Following previous studies $[18,19,40]$, the differences of network characteristics between male and female participants were examined, using the Network Comparison Test (NCT), a permutation test that assessed the difference between two networks (e.g., male participants vs. female participants) [41]. The NCT was performed on subsamples defined by gender using 1000 permutations as recommended previously $[18,42]$. This procedure assessed the global network strength by comparing the absolute sum of all edge weights between the networks. Next, the distributions of edge weights were compared within each network in order to characterize the structure of the network. Finally, the differences in strength for each edge were compared between the two networks after controlling for multiple tests (Holm-Bonferroni correction of $p$ values). All the tests were performed with the R-package "NetworkComparisonTest" 2.0.1 [43].

\section{RESULTS \\ Descriptive statistics}

A total of 11072 participants fulfilled the study entry criteria and were included in this study, with 2105 males and 8815 females. Table 1 shows the basic socio-demographic characteristics of the participants. Mean, SD, skewness, and kurtosis, of depressive symptoms measured by the PHQ-9 are shown in Table 2. The mean (SD) of PHQ-9 total score was $0.66(0.22)$ after they were 
transformed into binary variables for the Ising model. The items of Energy and Anhedonia had the highest mean ratings, while the symptoms of Suicide thoughts and Motor problems had the lowest mean ratings.

\section{Network structure and centrality measures analysis}

Following previous studies $[18,44]$, item informativeness (i.e., SD of the item) and item redundancy were checked first. We found that no item was poorly informative (i.e., $2.5 \mathrm{SD}$ below the mean level of informativeness [18], $M_{\mathrm{SD}}=0.43 \pm 0.07$ ) and no item was redundant with any other item (i.e., $<25 \%$ of statistically different correlations). Therefore, all the PHQ-9 items were included in the analyses.

The network of depressive symptoms, as estimated by the Ising model, is shown in Fig. 1. Several nodes were highly connected with the rest of the network, including Guilt (item 6), Sad Mood (item 2) and Energy (item 4). Moreover, there were strong positive correlations between Anhedonia-Sad Mood, Guilty-Suicide, Concentration-Motor, Energy-Appetite, Sad Mood-Guilty, and SleepEnergy. A weighted adjacency matrix was used to examine the

Table 1. Socio-demographic characteristics of the study population $(N=11072)$.

\begin{tabular}{|c|c|c|}
\hline Variables & $N$ & $\%$ \\
\hline \multicolumn{3}{|l|}{ Gender $^{a}$} \\
\hline Men & 2105 & 19.0 \\
\hline Women & 8815 & 79.6 \\
\hline Married/cohabiting & 5933 & 53.6 \\
\hline \multicolumn{3}{|l|}{ Education level $^{\mathrm{a}}$} \\
\hline Elementary or below ${ }^{b}$ & 25 & 0.2 \\
\hline High school & 2668 & 24.1 \\
\hline College or higher & 8244 & 74.5 \\
\hline Low income ${ }^{c}$ & 1289 & 11.6 \\
\hline \multirow[t]{2}{*}{ Living alone } & 399 & 3.6 \\
\hline & Mean & SD \\
\hline Age (years) & 39.07 & 8.83 \\
\hline PHQ-9 total score & 9.60 & 6.04 \\
\hline \multicolumn{3}{|c|}{$\begin{array}{l}\text { SD standard deviation, } P H Q-9 \text { the } 9 \text {-item Patient Health Questionnaire. } \\
\text { a There are missing values, therefore, the total percentage is not equal } \\
\text { to } 100 \% \text {. } \\
\text { b Low income = low household's monthly income ( }<100 \text { hundred HKD } \approx \\
1288 \text { dollar). } \\
{ }^{c} \text { Elementary or below = less than } 7 \text { years of education. }\end{array}$} \\
\hline
\end{tabular}

numerical interactions between these symptoms (Supplementary Table 1). Fig. 2 illustrated centrality measures (i.e., strength, betweenness, and closeness) of all the symptoms within the network. The symptom Guilt showed the highest strength, betweenness, and closeness, followed by Sad Mood and Energy.

\section{Network accuracy and stability}

The edge weights in the current sample were consistent with the bootstrapped sample, especially the connections with larger weights,

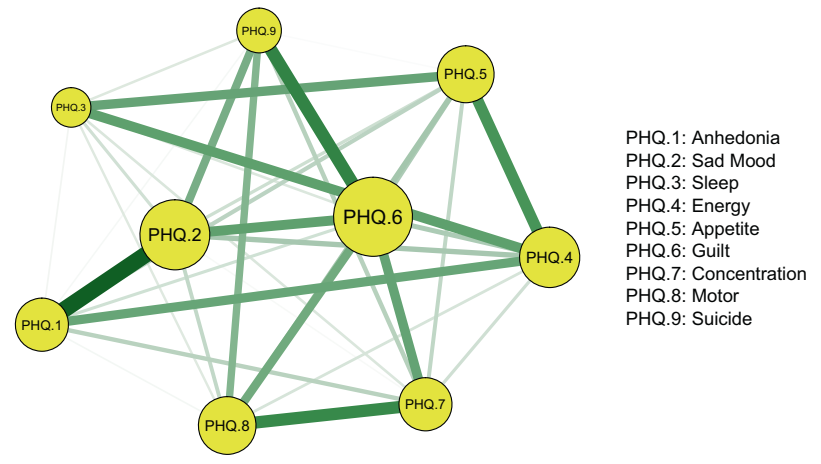

Fig. 1 Estimated network model for dichotomized depressive symptoms in the total sample. The network model was estimated using the Ising model.

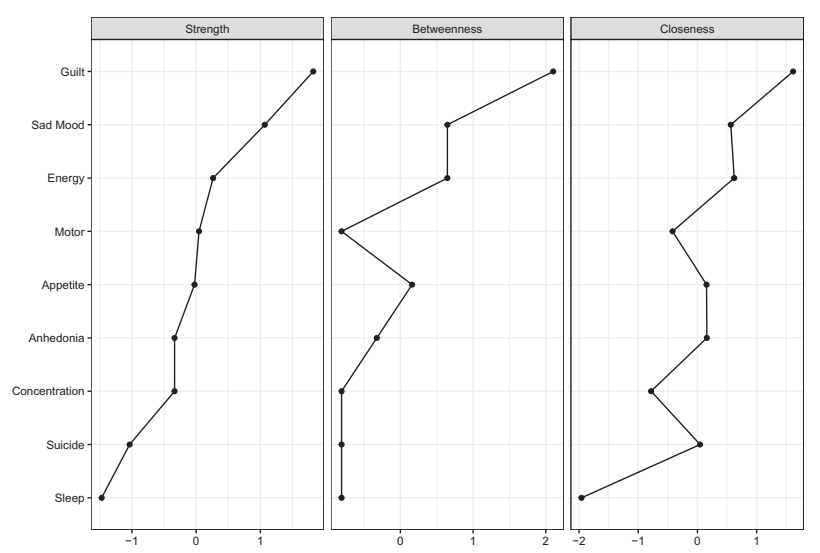

Fig. 2 Centrality measures of all symptoms within the network. The figure shows centrality measure (i.e., strength, betweenness, and closeness) of all factors within the network (z-scores).

Table 2. Mean, standard deviation, minimum, maximum, skewness, and kurtosis, and frequency of depressive symptoms as measured by the PHQ-9 $(N=11072)$.

\begin{tabular}{llllllllll} 
Depressive symptoms & PHQ-9 item & $\boldsymbol{M}$ & SD & Min & Max & Skewness & Kurtosis & \% (Absence) & \% (Presence) \\
\hline Anhedonia & 1 & 0.86 & 0.34 & 0 & 1 & -2.11 & 2.47 & 13.7 & 86.3 \\
\hline Sad Mood & 2 & 0.82 & 0.39 & 0 & 1 & -1.64 & 0.68 & 18.3 & 81.7 \\
\hline Sleep & 3 & 0.79 & 0.41 & 0 & 1 & -1.4 & -0.04 & 21.3 & 78.7 \\
\hline Energy & 4 & 0.87 & 0.33 & 0 & 1 & -2.23 & 2.95 & 12.8 & 87.2 \\
\hline Appetite & 5 & 0.72 & 0.45 & 0 & 1 & -0.98 & -1.04 & 28.0 & 72.0 \\
\hline Guilt & 6 & 0.56 & 0.5 & 0 & 1 & -0.24 & -1.94 & 44.0 & 56.0 \\
\hline Concentration & 7 & 0.62 & 0.49 & 0 & 1 & -0.49 & -1.76 & 38.0 & 62.0 \\
\hline Motor & 8 & 0.47 & 0.5 & 0 & 1 & 0.11 & -1.99 & 52.8 & 47.2 \\
\hline Suicide & 9 & 0.22 & 0.42 & 0 & 1 & 1.32 & -0.26 & 77.5 & 22.5
\end{tabular}

$M$ mean, Min minimum, Max maximum, PHQ-9 The Patient Health Questionnaire-9, SD standard deviation. 


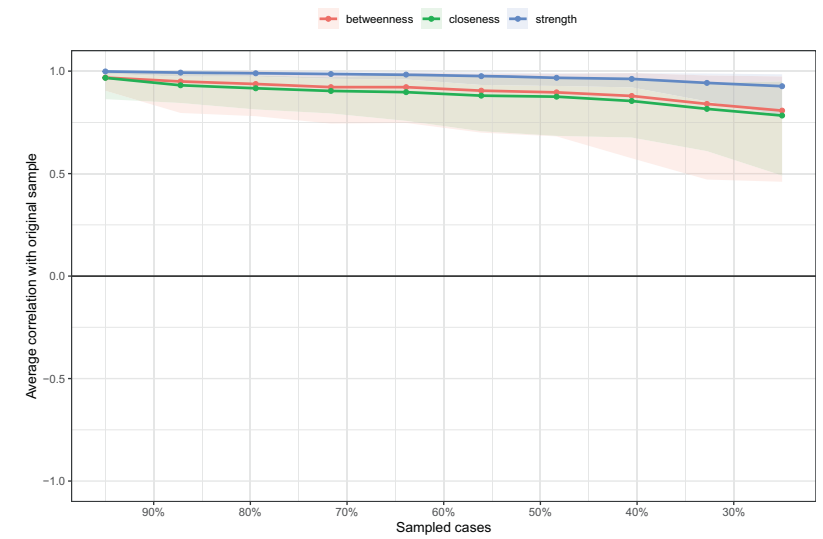

Fig. 3 Stability of centrality indices by case dropping subset bootstrap. The $x$-axis represents the percentage of cases of the original sample used at each step. The $y$-axis represents the average of correlations between the centrality indices from the original network and the centrality indices from the networks that were re-estimated after excluding increasing percentages of cases. Each line indicates the correlations among betweenness, closeness, and strength.

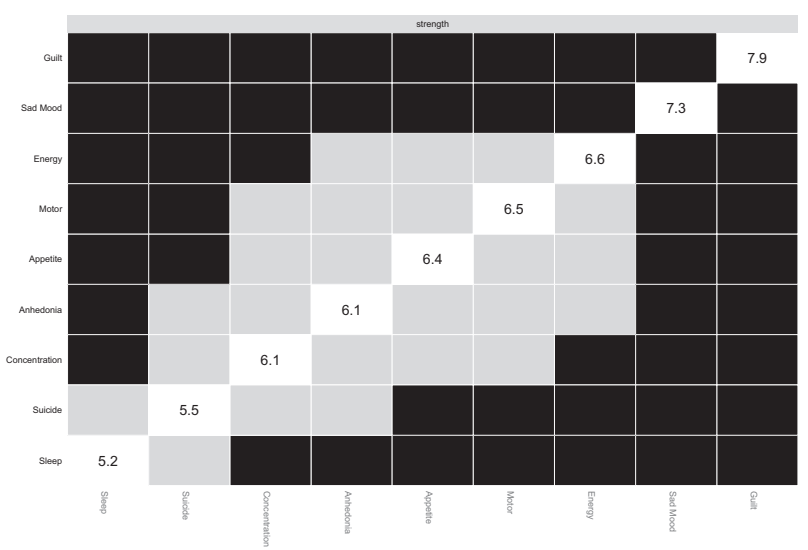

Fig. 4 Estimation of node strength difference by bootstrapped difference test. Bootstrapped difference tests between node strength of factors. Gray boxes indicate nodes that do not significantly differ from one-another. Black boxes represent nodes that differ significantly from one another $(\alpha=0.05)$. White boxes show the values of node strength.

indicating that the current network structure was stable (Supplementary Fig. 1). The case-dropping subset bootstrap procedure showed that the values of betweenness, closeness and strength remained stable even after dropping large proportions of the sample (Fig. 3). Although betweenness reported slightly low stability (i.e., CS-C = 0.52 ), closeness showed higher stability (i.e., $C S-C=0.59$ ) compared to the primary one. In contrast, the strength index in this sample was robust and trustworthy (i.e., $\mathrm{CS}-\mathrm{C}=0.75$; i.e., after dropping up to $75 \%$ of the sample, the order of the symptoms in strength was still correlated with the original one $(r=0.7))$. Hence, we focused on the interpretation of symptom strength based on this network analysis.

In terms of strength, Guilt, and Sad Mood were statistically stronger compared to other symptoms. Energy, Motor and Appetite appeared stronger than other symptoms in the network (Fig. 4). The bootstrapped difference tests also revealed that a large proportion of the comparisons among edge weights were statistically significant (Supplementary Fig. 2).
Symptom mean levels, variability, and association with strength centrality index

In the whole sample, depressive symptoms with the highest mean levels were Energy, Anhedonia, Sad Mood and Sleep (Table 2). However, the mean PHQ-9 symptom level was not related to the symptom strength $\left(r_{s}=0.15\right)$, while symptom standard deviation was also not correlated to the symptom strength $\left(r_{s}=0.21\right.$, which indicates that high symptom centrality was not related to the mean level of the symptoms and their variability.

\section{Network and symptom mean levels comparisons between the two genders}

We compared the network models and network centrality indices between male ( $n=2$ 105) and female participants $(n=8815)$ (Fig. 5 and Supplementary Fig. 3). There were no significant gender differences in network global strength (males: 3.81 vs. females: 3.77 ; global strength difference $=0.03, p=0.259$ ), distribution of edge weights $(M=0.06, p=0.73)$ and individual edge weights (all $p$ values $>0.05$ after Holm-Bonferroni corrections) (Supplementary Fig. 4).

In contrast, there were gender differences in mean levels of certain individual symptoms (Supplementary Table 2). Female participants reported significantly higher mean levels of Sleep problem and Appetite $(P<0.01)$, while male participants reported higher mean levels of Sad Mood, Guilt, Motor, and Suicide thoughts $(P<0.01)$. Similar to the findings obtained in the whole sample, symptom strength was not related to items' variability in both female $\left(r_{s}=0.22\right)$ and male $\left(r_{s}=0.21\right)$ participants. Strength was not correlated to symptoms mean levels in either gender $\left(r_{s}=\right.$ 0.12 in female and $r_{s}=0.25$ in male participants), indicating that network symptoms strength and mean levels of symptoms were independent between male and female samples.

\section{Covariates (age, education level, and marital status)}

Previous studies found that age, education level and marital status were associated with the epidemiology and clinical features of depression [20]. Hence, following previous studies [19], the network model and the local structure indexes were reestimated, after controlling for age, education level, and marital status as covariates. Compared to the original network model, an almost identical network was obtained with respect to the magnitude of edges $(r=0.995,95 \% \mathrm{Cl}=[0.991 ; 0.997], P<0.01)$, and strength $(r=0.984,95 \% \mathrm{Cl}=[0.931 ; 0.997], P<0.01))$ (Supplementary Fig. 5).

\section{DISCUSSION}

To the best of our knowledge, this was the first study to characterize the network structure of depressive symptoms among the general population in Hong Kong. Guilt was the most central symptom in the network of depression, followed by Sad Mood and Energy symptoms that could maintain or trigger the remaining depressive symptoms in this sample.

Guilt was the most central symptom in Hong Kong residents during the COVID-19 pandemic. Guilt is an interpersonally driven emotion arising from the belief that an individual has hurt others. Due to this belief, guilt is often accompanied by regret, remorse, or worries over a transgression leading to depressive states [45]. Guilt may manifest as self-blame, worthlessness, powerlessness, inferiority [46], hopelessness and helplessness [47], which could lead to development of depressive symptoms [46]. Energy is another central symptom in this study, which is consistent with the findings of a previous drug trial of duloxetine, with energy improvement as a major outcome measure [48]. A previous study [49] compared gender differences in the associations between individual symptoms of major depression and other psychiatric disorders with network analysis using the data extracted from the 2011 and 2016 Korean Epidemiologic Catchment Area Study $(n=$ 
Male

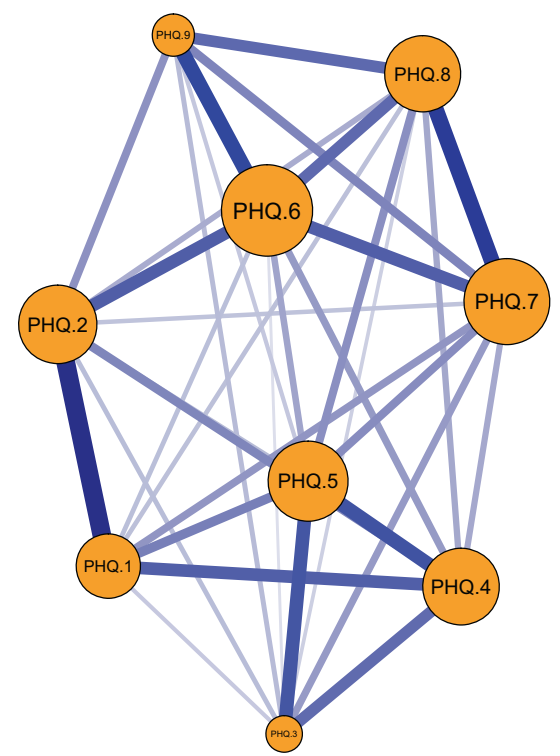

Female

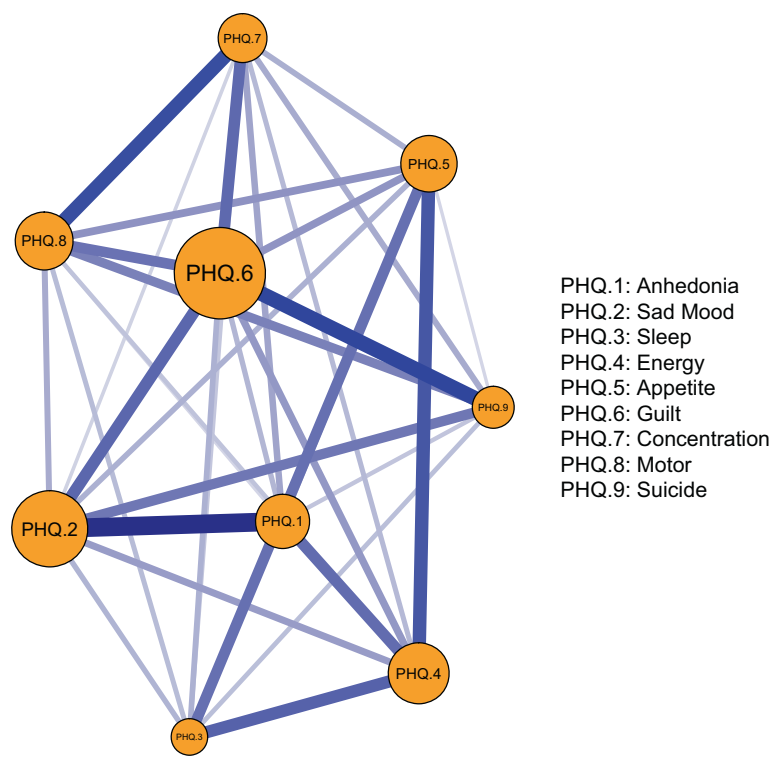

Fig. 5 Estimated network models for dichotomized depressive symptoms in male and female participants. Left panel: network structure in male participants ( $n=2$ 105); Right panel: network structure in female participants $(n=8815)$.

907), and found that the global strength did not differ between genders, which is consistent with our findings.

Sad Mood is another central depressive symptom in the network of Hong Kong residents, which is consistent with the Beard et al.'s finding [50] that 'sad mood' and 'too much worry' were the most central to the network of depressive symptoms as measured by the PHQ-9 and the 7-item Generalized Anxiety Disorder Scale (GAD-7). However, Beard et al.'s study used network analysis to investigate the depression and anxiety symptom relationships on psychiatric inpatients in the US, whilst our study investigated depressive symptoms in a community sample. Despite different study samples, sad mood is the hallmark symptom required for meeting the diagnosis of major depression [51]. Additionally, our findings are similar to another study using network model with the PHQ-9 in the German general population [52], in which sad mood, energy loss and guilt were central depressive symptoms. In another study using network analysis on depression and anxiety symptom relations as measured by the PHQ-9 and GAD-7 in a psychiatric sample [50], "sad mood" was the most central symptom, followed by certain anxiety symptoms such as "too much worry", "unable to control worry", and "unable to relax"; furthermore, the most central symptoms in the depression community were low energy, anhedonia, and guilt, which partly supports our findings. In another study on the changes in network centrality of psychiatric symptoms as measured by the GAD-7 and PHQ-9 between the COVID-19 outbreak and after peak, "loss of energy" played an important role in the network model [42].

The NCT did not reveal significant gender differences in the network structures of depressive symptoms, which is consistent with the findings of previous network analyses [18, 19,40], but does not directly support the notion that there are different clinical features between the two genders [53-57]. Epidemiological studies found significant gender differences in depression prevalence [53], with higher rates of depression in women. A systematic review revealed a female preponderance of 1.5-3 times higher depression rates [55]. For clinical features of depression, the $\operatorname{STAR}^{*} \mathrm{D}$ study showed that appetite and weight changes, anergia, psychomotor agitation and sympathetic arousal symptoms were usually over-represented in women [58]. In another study of twins with depression, female twins usually experienced more severe fatigue, hypersomnia and psychomotor retardation, while male twins experienced more severe insomnia and agitation [59]. However, in the current network analysis, no significant gender differences in the network structures of depressive symptoms were found. The different results between this and the abovementioned studies could be explained by several reasons. First, most studies on epidemiology and clinical features of depression were based on total score of standardized scales on depression such as the PHQ9, which however has the potential to obscure the important differences between individual symptoms and the relationships among symptoms [50]. In contrast, the network approach assesses interactions between individual symptoms [6]. Second, considering that disproportional gender ratio (Male $=2105$ VS. Female $=8$ 815) in the current sample, the findings on gender differences in this study are preliminary. Finally, it is possible that the gender differences in network analysis may be partly masked by the negative impact of the pandemic on mental health, as both genders may be equally affected by fears about the pandemic and related consequences and public health measures such as lockdown.

In the past years Hong Kong has been confronted by repeated cooccurrence of population-level stressors including social unrest (e.g., the Occupy Central Movement (Umbrella Movement) in 2014; antiextradition bill in 2019) and public health crises (e.g., COVID-19 pandemic in 2019), all of which were associated with increased risk of mental problems including depression. For instance, some studies $[60,61]$ examined the association between the Occupy Central Movement and population mental distress using anonymous random population-based telephone survey, and results showed that young age (of those who did not participate in the movement), worry about safety, negative emotional responses to media reports and local political situations, conflicts with peers about this movement were significantly associated with mental distress.

In 2019, a series of local protests against the extradition bill progressed from initial political protests to violent protests in Hong Kong, ended up with thousands of arrests [62]. This social unrest has brought about tremendous mental distress to Hong Kong residents $[63,64]$. The subsequent outbreak of COVID-19 in early 2020 has made the matters even worse as this pandemic has led to widespread panic in Hong Kong. The rapid transmission of this novel coronavirus locally and globally, in the absence of effective treatment/vaccine, alongside insufficient personal 
protective equipment (e.g., face-masks) and conflictual messages by the local government on quarantine, social distancing and contingent public health preventive measures (closure of major theme parks, entertainment amenities, sports gymnasiums, suspension of face-to-face teaching/learning in all educational institutions) have reminded the lay public their traumatic memories during the 2003 SARS epidemic [65].

At the time of report, Hong Kong is in the fourth wave of the COVID-19 pandemic. This long-haul battle combating against the pandemic, alongside with recurrent political instability may cause unease, anxiety and depression in the general population [66]. For example, Wong et al [67]. examined the impact of repeated exposure to social unrest-related traumatic events and COVID-19, pandemicrelated events on Hong Kong residents and found that rumination, stressful life events and pandemic-related events were significant predictors (all $P<0.001$ ) of depression. All subtypes of ruminations were significant mediators [67], which is consistent with our finding that guilt is the key central depressive symptom in this study.

Due to the recent social unrest and public health crises that happened in Hong Kong, rumination (guilt) is the central depressive symptoms in this network analysis. Nevertheless, previous studies predominantly focused on depressive rumination [68] but rarely examined rumination relating to external societal and environmental situations, although such rumination may have a substantial long-term impact on population mental health leading to increased psychiatric morbidities (particularly depression) and global disease burden. A 10-year cohort study [63] on mental health burden and associated factors of depression and posttraumatic stress disorder (PTSD) found that the weighted prevalence of depression among Hong Kong adults was $1.9 \%$ (95\% Cl: 1.6-2.1\%) at baseline (between 2009 and 2014). In contrast, the weighted prevalence of depressive symptoms increased to $37.4 \%(95 \% \mathrm{Cl}: 35.1-39.7 \%)$, with $11.2 \%(95 \% \mathrm{Cl}$ : 9.8-12.7\%) having probable depression in 2019-2020.

The strengths of this study include the large sample size and the homogenous study sample. However, several implications needed to be addressed. First, this is a cross-sectional study, therefore the causality and the dynamic relationships between variables cannot be estimated. Second, this study was conducted in a community adult sample, hence, the findings cannot be generalized to special populations such as adolescents, the elderly, and patients with major depression. Third, since patterns and clinical features of depression are greatly determined by socioeconomic contexts $[20,21,64]$, the structure of depressive symptoms in settings with different socioeconomic and cultural contexts should be examined separately. Fourth, depressive symptoms were assessed by PHQ-9 rather than clinical interview; therefore, atypical features of depression could not be identified, which may bias the results to an uncertain extent. Finally, the possibility that some participants suffered from depressive disorders could not be excluded, which may bias the findings to an uncertain extent.

In conclusion, this network analysis revealed that Guilt, Sad Mood and Energy were the most central symptoms of depression in this study. These three central symptoms constitute the "backbone" that sustained the depressive symptom structure among Hong Kong residents during the COVID-19 pandemic, which provided a unique opportunity to understand the interactions between prolonged social unrest and recurrent public health crisis may contribute to depressive symptoms among Hong Kong residents amidst COVID-19 pandemic. Timely mental health treatment to reduce rumination, such as cognitive behavioral therapy (CBT), was crucial in the prevention of further exacerbation of depressive symptoms among Hong Kong residents especially during the pandemic era and beyond.

\section{CODE AVAILABILITY}

Analytic code for this work is available upon request.

\section{REFERENCES}

1. American Psychiatric Association. Diagnostic and statistical manual of mental disorders. 5th ed. Vol. 21 (2013)

2. Foley DL, Goldston DB, Costello EJ, Angold A. Proximal psychiatric risk factors for suicidality in youth: the Great Smoky Mountains Study. Arch Gen Psychiatry. 2006;63:1017-24.

3. Fröjd SA, Nissinen ES, Pelkonen MU, Marttunen MJ, Koivisto AM, Kaltiala-Heino R. Depression and school performance in middle adolescent boys and girls. J Adolescence. 2008;31:485-98.

4. Buysse DJ, Angst J, Gamma A, Ajdacic V, Eich D, Rössler W. Prevalence, course, and comorbidity of insomnia and depression in young adults. Sleep 2008;31:473-80.

5. Lynch FL, Clarke GN. Estimating the economic burden of depression in children and adolescents. Am J Preventive Med. 2006;31:143-51.

6. Borsboom D. Psychometric perspectives on diagnostic systems. J Clin Psychol. 2008;64:1089-108.

7. Wakefield JC. The concept of mental disorder: diagnostic implications of the harmful dysfunction analysis. World Psychiatry. 2007;6:149-56.

8. Fried El. Problematic assumptions have slowed down depression research: why symptoms, not syndromes are the way forward. Front Psychol. 2015;6:309.

9. Fried El, Nesse RM. The impact of individual depressive symptoms on impairment of psychosocial functioning. PLoS ONE. 2014;9:e90311.

10. Aan Het Rot M, Mathew SJ, Charney DS. Neurobiological mechanisms in major depressive disorder. Cmaj Open. 2009;180:305-13.

11. Berlim MT, Richard-Devantoy S, Dos Santos NR, Turecki G. The network structure of core depressive symptom-domains in major depressive disorder following antidepressant treatment: a randomized clinical trial. Psychol Med. 2020;1-15. https://doi.org/10.1017/S0033291720001002.

12. Borsboom D. A network theory of mental disorders. World Psychiatry. 2017;16:5-13.

13. Borsboom D, Cramer AO. Network analysis: an integrative approach to the structure of psychopathology. Annu Rev Clin Psychol. 2013;9:91-121.

14. Cramer AO, Borsboom D, Aggen SH, Kendler KS. The pathoplasticity of dysphoric episodes: differential impact of stressful life events on the pattern of depressive symptom inter-correlations. Psychol Med. 2012;42:957-65.

15. Garabiles MR, Lao CK, Wang S, Hall BJ. The network structure of posttraumatic stress disorder among Filipina migrant domestic workers: comorbidity with depression. Eur J Psychotraumatol. 2020;11:1765544.

16. Garabiles MR, Lao CK, Xiong Y, Hall BJ. Exploring comorbidity between anxiety and depression among migrant Filipino domestic workers: a network approach. J Affect Disord. 2019;250:85-93.

17. Cramer AO, Waldorp LJ, van der Maas HL, Borsboom D. Comorbidity: a network perspective. Behav Brain Sci. 2010;33:137-50. discussion 150-193

18. Mullarkey MC, Marchetti I, Beevers CG. Using network analysis to identify central symptoms of adolescent depression. J Clin Child Adolesc Psychol. 2019;48:656-68.

19. Belvederi Murri M, Amore M, Respino M, Alexopoulos GS. The symptom network structure of depressive symptoms in late-life: results from a European population study. Mol Psychiatry. 2020;25:1447-56.

20. Kleinman A. Culture and depression. N Engl J Med. 2004;351:951-3.

21. Compton WM, Conway KP, Stinson FS, Grant BF. Changes in the prevalence of major depression and comorbid substance use disorders in the United States between 1991-1992 and 2001-2002. Am J Psychiatry. 2006;163:2141-7.

22. Xiang YT, Yang Y, Li W, Zhang L, Zhang Q, Cheung T, et al. Timely mental health care for the 2019 novel coronavirus outbreak is urgently needed. Lancet Psychiatry. 2020;7:228-9.

23. Kroenke K, Spitzer RL, Williams JB. The PHQ-9: validity of a brief depression severity measure. J Gen Intern Med. 2001;16:606-13.

24. Löwe B, Kroenke K, Herzog W, Gräfe K. Measuring depression outcome with a brief self-report instrument: sensitivity to change of the Patient Health Questionnaire (PHQ-9). J Affect Disord. 2004;81:61-66.

25. Wang W, Bian Q, Zhao Y, Li X, Wang W, Du J, et al. Reliability and validity of the Chinese version of the Patient Health Questionnaire (PHQ-9) in the general population. Gen Hosp Psychiatry. 2014;36:539-44.

26. Zhang YL, Liang W, Chen ZM, Zhang HM, Zhang JH, Weng XQ, et al. Validity and reliability of Patient Health Questionnaire-9 and Patient Health Questionnaire-2 to screen for depression among college students in China. Asia Pac Psychiatry. 2013;5:268-75.

27. Yu X, Tam WW, Wong PT, Lam TH, Stewart SM. The Patient Health Questionnaire9 for measuring depressive symptoms among the general population in Hong Kong. Compr Psychiatry. 2012;53:95-102.

28. van Borkulo CD, Borsboom D, Epskamp S, Blanken TF, Boschloo L, Schoevers RA, et al. A new method for constructing networks from binary data. Sci Rep. 2014;4:5918.

29. Epskamp S, Cramer AO, Waldorp U, Schmittmann VD, Borsboom D. qgraph: Network visualizations of relationships in psychometric data. J Stat Softw. 2012;48:1-18.

30. Barber RF, Drton M. High-dimensional Ising model selection with Bayesian information criteria. Electron J Stat. 2015;9:567-607. 
31. Ravikumar $P$, Wainwright MJ, Lafferty JD. High-dimensional Ising model selection using $\ell 1$-regularized logistic regression. Ann Stat. 2010;38:1287-319.

32. Foygel R, Drton M. Extended Bayesian information criteria for Gaussian graphical models. Adv Neural Inf Process Syst. 2010;1:604-12.

33. Valente TW. Network interventions. Science 2012;337:49-53.

34. Opsahl T, Agneessens F, Skvoretz J. Node centrality in weighted networks: Generalizing degree and shortest paths. Soc Netw. 2010;32:245-51.

35. R Core Team. R: A language and environmentfor statistical computing. 2020. https://www.R-project.org/.

36. Epskamp S, Borsboom D, Fried El. Estimating psychological networks and their accuracy: a tutorial paper. Behav Res Methods. 2018;50:195-212.

37. Chernick MR. Bootstrap methods: a guide for practitioners and researchers. 619 . John Wiley \& Sons; 2011.

38. Costenbader E, Valente TW. The stability of centrality measures when networks are sampled. Soc Netw. 2003;25:283-307.

39. Epskamp S, Fried El. Package 'bootnet'. 2018. https://cran.r-project.org/web/ packages/bootnet/index.html.

40. Mullarkey, MC, Stewart, RA, Wells, TT, Shumake, J \& Beevers, CG. Self-Dislike and Sadness are Central Symptoms of Depression in College Students: A Network Analysis. https://doi.org/10.31234/osf.io/fujmb. (2018).

41. van Borkulo C, Boschloo L, Borsboom D, Penninx BW, Waldorp LJ, Schoevers RA. Association of symptom network structure with the course of depression. Jama Psychiatry. 2015;72:1219-26.

42. Wang Y, Hu Z, Feng Y, Wilson A, Chen R. Changes in network centrality of psychopathology symptoms between the COVID-19 outbreak and after peak. Mol Psychiatry. 2020;25:3140-9.

43. van Borkulo C, Epskamp S, Jones P, Haslbeck J, Millner A. Package 'NetworkComparisonTest'. 2016. https://cran.r-project.org/web/packages/NetworkComparisonTest/ index.html.

44. Marchetti I. Hopelessness: a network analysis. Cogn Ther Res. 2019;43:611-9.

45. Kim S, Thibodeau R, Jorgensen RS. Shame, guilt, and depressive symptoms: a meta-analytic review. Psychol Bull. 2011;137:68-96.

46. Gambin M, Sharp C. The relations between empathy, guilt, shame and depression in inpatient adolescents. J Affect Disord. 2018;241:381-7.

47. Bademci HÖ, Karadayı EF, Pur Karabulut IG, Warfa N. Who is the helper? Who is being helped? The benefits of psychosocial support to correctional officers in Turkey. Psychodyn Pr. 2016;22:351-65.

48. Harada E, Kato M, Fujikoshi S, Wohlreich MM, Berggren L, Tokuoka H. Changes in energy during treatment of depression: an analysis of duloxetine in double-blind placebo-controlled trials. Int J Clin Pr. 2015;69:1139-48.

49. Shim EJ, Ha H, Park JE, Kim BS, Chang SM, Hong JP, et al. Gender-based examination of the association between individual symptoms of alcohol use disorder, major depressive disorder, and suicidal behaviors: a network analysis of data from the Korean Epidemiologic Catchment Area Study. J Affect Disord. 2020;272:432-9

50. Beard C, Millner AJ, Forgeard MJ, Fried El, Hsu KJ, Treadway MT, et al. Network analysis of depression and anxiety symptom relationships in a psychiatric sample. Psychol Med. 2016;46:3359-69.

51. Fried El, Epskamp S, Nesse RM, Tuerlinckx F, Borsboom D. What are 'good' depression symptoms? Comparing the centrality of DSM and non-DSM symptoms of depression in a network analysis. J Affect Disord. 2016;189:314-20.

52. Hartung TJ, Fried El, Mehnert A, Hinz A, Vehling S. Frequency and network analysis of depressive symptoms in patients with cancer compared to the general population. J Affect Disord. 2019;256:295-301.

53. Angst J, Gamma A, Gastpar M, Lépine JP, Mendlewicz J, Tylee A, et al. Gender differences in depression. Eur Arch Psychiatry Clin Neurosci. 2002;252:201-9.

54. Nolen-Hoeksema S. Gender differences in depression. Curr Dir Psychol Sci. 2001;22:173-6.

55. Salk RH, Hyde JS, Abramson LY. Gender differences in depression in representative national samples: meta-analyses of diagnoses and symptoms. Psychol Bull. 2017;143:783-822.

56. Piccinelli M, Wilkinson G. Gender differences in depression. Critical review. Rev Br J Psychiatry. 2000;177:486-92.

57. Parker G, Fletcher K, Paterson A, Anderson J, Hong M. Gender differences in depression severity and symptoms across depressive sub-types. J Affect Disord. 2014;167:351-7.

58. Marcus SM, Young EA, Kerber KB, Kornstein S, Farabaugh AH, Mitchell J, et al. Gender differences in depression: findings from the STAR*D study. J Affect Disord. 2005;87:141-50.

59. Khan AA, Gardner CO, Prescott CA, Kendler KS. Gender differences in the symptoms of major depression in opposite-sex dizygotic twin pairs. Am J Psychiatry 2002;159:1427-9.

60. Lau JTF, Kim Y, Wu A, Wang Z, Huang B, Mo P. The Occupy Central (Umbrella) movement and mental health distress in the Hong Kong general public: political movements and concerns as potential structural risk factors of population mental health. Soc Psychiatry Psychiatr Epidemiol. 2017:52:525-36.

61. Hou WK, Hall BJ, Canetti D, Lau KM, Ng SM, Hobfoll SE. Threat to democracy: physical and mental health impact of democracy movement in Hong Kong. J Affect Disord. 2015;186:74-82.

62. Shek DTL. Protests in Hong Kong (2019-2020): a perspective based on quality of life and well-being. Appl Res Qual Life. 2020;13:1-17.

63. Ni MY, Kim Y, McDowell I, Wong S, Qiu H, Wong IO, et al. Mental health during and after protests, riots and revolutions: a systematic review. Aust N. Z J Psychiatry. 2020;54:232-43.

64. Hou WK, Hall BJ, Liang L, Li TW, Liu H, Galea S. Probable depression and suicidal ideation in Hong Kong amid massive civil unrest. Ann Epidemiol. 2021;54:45-51.

65. Cheung T, Fong TKH, Bressington D. COVID-19 under the SARS Cloud: Mental Health Nursing during the Pandemic in Hong Kong. J Psychiatr Ment Health Nurs. 2020;28:115-7.

66. Cheung T, Lam SC, Lee PH, Xiang YT, Yip PSF. Global imperative of suicidal ideation in 10 countries amid the COVID-19 pandemic. Front Psychiatry. 2020;11:588781.

67. Wong SMY, Hui C, Wong C, Suen YN, Chan S, Lee E, et al. Mental Health Risks after Repeated Exposure to Multiple Stressful Events during Ongoing Social Unrest and Pandemic in Hong Kong: The Role of Rumination: Risques pour la santé mentale après une exposition répétée à de multiples événements stressants d'agitation sociale durable et de pandémie à Hong Kong: le rôle de la rumination. Can J Psychiatry. 2020;66:577-85.

68. Nolen-Hoeksema S, Wisco BE, Lyubomirsky S. Rethinking rumination. Perspect Psychol Sci. 2008;3:400-24.

\section{ACKNOWLEDGEMENTS}

The authors are grateful to our research assistants and trained student helpers who assisted in the data collection process. We also thanked Rui Liu, Qinge Zhang, Ling Zhang in Beijing Anding Hospital for their contribution to data analyses, and we are grateful to all the participants who participated in this study.

\section{AUTHOR CONTRIBUTIONS}

Study design: T.C., Y.-T.X. Data collection, analysis and interpretation: S.L., Y.J. Drafting of the manuscript: T.C., Y.J., Y.-T.X. Critical revision of the manuscript: B.J.H. Approval of the final version for publication: all co-authors.

\section{COMPETING INTERESTS}

The authors declare no competing interests.

\section{ADDITIONAL INFORMATION}

Supplementary information The online version contains supplementary material available at https://doi.org/10.1038/s41398-021-01543-z.

Correspondence and requests for materials should be addressed to Teris Cheung or Yu-Tao Xiang

Reprints and permission information is available at http://www.nature.com/ reprints

Publisher's note Springer Nature remains neutral with regard to jurisdictional claims in published maps and institutional affiliations.

cc) (i)

Open Access This article is licensed under a Creative Commons Attribution 4.0 International License, which permits use, sharing, adaptation, distribution and reproduction in any medium or format, as long as you give appropriate credit to the original author(s) and the source, provide a link to the Creative Commons license, and indicate if changes were made. The images or other third party material in this article are included in the article's Creative Commons license, unless indicated otherwise in a credit line to the material. If material is not included in the article's Creative Commons license and your intended use is not permitted by statutory regulation or exceeds the permitted use, you will need to obtain permission directly from the copyright holder. To view a copy of this license, visit http://creativecommons. org/licenses/by/4.0/.

(c) The Author(s) 2021 
THE INTERNATIONAL RESEARCH COLLABORATION ON COVID-19

Lorna Kwai Ping Suen $\mathbb{D}^{9}$, Shun Chan ${ }^{10}$, Hilda Sze Wing HO $\mathbb{D}^{11}$, Kin Bong Hubert Lam (D) ${ }^{12}$, Emma Yun-zhi Huang $\mathbb{D}^{13}$, Ying Xiao ${ }^{14}$, Fernanda Maria Vieira Pereira-Ávila (iD) ${ }^{15}$, Elucir Gir (iD) ${ }^{16}$, Menevse Yildirim (iD) ${ }^{17}$, Seyda Seren Intepeler (iD) ${ }^{18}$, Tella Lantta (iD) ${ }^{19}$, Kyungmi Lee $\mathbb{i D}^{20}$, Nayeon Shin $\mathbb{1 D}^{21}$, Laurence Lloyd Parial $\mathbb{1 D}^{22}$, Tor Michael Rossing ${ }^{23}$, Ching Yuk Hon ${ }^{22}$, Merissa Tsang ${ }^{24}$, Jessica P. Braz Poeys $\mathbb{i D}^{25}$ and Tommy Kwan Hin Fong ${ }^{26}$

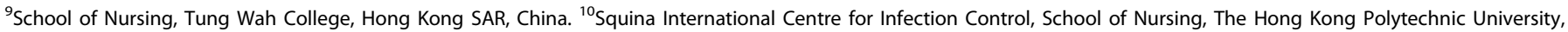

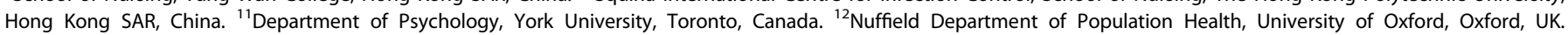

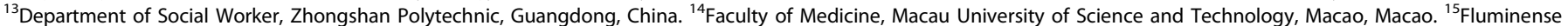

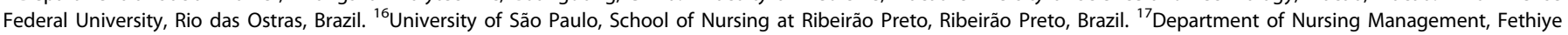

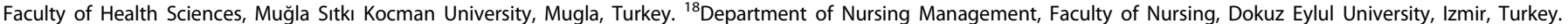

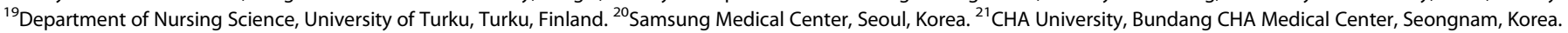

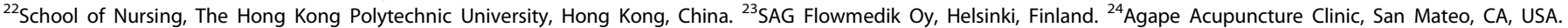

${ }^{25}$ Westways Staffing Inc, Austin, TX, USA. ${ }^{26}$ School of Nursing, The Hong Kong Polytechnic University, Hong Kong SAR, China. 